\title{
Macroscopic Findings Domain
}

National Cancer Institute

\section{Source}

National Cancer Institute. Macroscopic Findings Domain. NCI Thesaurus. Code C95094.

A subject domain utilized for the submission of information encompassing and representing data, vocabulary or records related to macroscopic findings. 